\title{
The Design and Implementation of General Symmetrical Self-Organizing Map Decoder
}

\author{
Wei Xiao ${ }^{1,2, *}$ and Yong $\mathrm{Ai}^{1}$ \\ ${ }^{1}$ Electronic Information School, Wuhan University, Wuhan, Hubei, 430072, P.R. China; ${ }^{2}$ School of Engineering, Tibet \\ University, Lhasa, Tibet, 850000, P.R. China
}

\begin{abstract}
Traditional error correcting decoder is designed according to specific algorithm, which is a reverse course of encoding. In this paper, a novel general neural network decoder is proposed in the form of symmetrical self-organizing map (SSOM), which can achieve decoding function to any error correcting codes. The SSOM decoder is tested by decoding low-density parity-check (LDPC) code. The performance comparison of SSOM decoder and traditional decoder is accomplished by simulation. Actual results show that the SSOM decoder can implement both learning and decoding in the same time regardless of any encoding rules. And higher possibility of the codeword emergence means greater probability of correct error correction. Compare to traditional error correcting decoder, it is easy to construct, more intelligent to different codeword sets, which has certain prospect in future communication channel coding field.
\end{abstract}

Keywords: Error correcting codes, error correcting decoder, low-density parity-check (LDPC) code, neural network, symmetrical self-organizing map (SSOM) decoder.

\section{INTRODUCTION}

At present, error-correcting code technology has become a good method to obtain the reliable and timely communication. Therefore, error correcting decoder is studied by many researchers all the time. Neural network, which is an intelligent technology, is adopted as a novel method utilized in decoding field.

Generally speaking, the study of neural network decoder can be divided into three types:

1. Adopting neural network as main architecture of the decoder, where the neural network has relationship with the encoding rules [1-4].

2. Adopting neural network as main architecture of the decoder, where the neural network has no relationship with the encoding rules [5].

3. Using neural network as supplementary role in the decoder [6-9].

Combining genetic algorithm and neural network, a genetic neural network decoder is proposed in [1], which is close to the traditional soft decision in error correction performance. However, the decoder adopts genetic algorithm as main architecture, where neural network only plays supplementary role. An artificial neural network decoder is proposed in [3], which is suitable for lower complexity neural network. And the decoder must be trained by the sample data beforehand.
A neural network turbo decoder is proposed in [5], which performance is slight inferior to the maximum a posteriori (MAP) decoder. Furthermore, the decoder should be trained by a set of sample sets generated by the encoder in advance, where learning and decoding cannot be achieve at the same time.

Based on encoding check matrix, a neural decoder based on multi-layer perceptron (MLP) is presented to decode LDPC codes by soft decision manner in [6]. However, this neural network decoder is constructed according to encoding check matrix, which is not a general intelligent decoder. A novel Vitrbi decoder using MLP is proposed in [7]. However, the MLP neural network is adopted as a pre-processing technique to predict the distortion from communication channel, rather than main architecture of the decoder.

To sum up, general neural network decoder, regardless of encoding rules, which can achieve both leaning and decoding at the same time, is rarely concerned by researchers.

\section{MAIN IDEA OF SSOM DECODER}

\subsection{The Main Architecture of SSOM Decoder}

The SSOM decoder is composed by symmetrical selforganizing map (SSOM), neural network controller and output decision controller (Fig. 1). SSOM is a novel neural network made up of two self-organizing maps, where the weight sets are symmetrical. It is composed of input layer, neuron layer and output layer. Input layer accepts codeword form communication channel. Neuron layer is a map of codeword stored in SSOM. Output layer exports ultimate result of the decoder. The modification of weight sets is implemented by neural network controller, which is adopted to activate neurons of SSOM. The ultimate decision of SSOM decoder is made by output decision controller. 


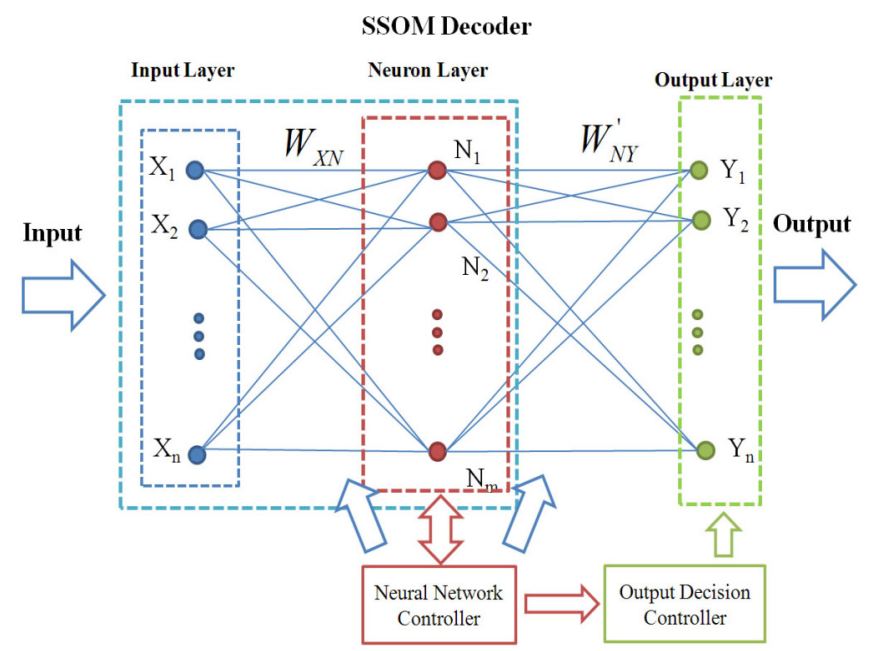

Fig. (1). The main architecture of SSOM decoder.

\subsection{Specific Algorithm Steps of SSOM Decoder}

There are several basic steps in implementation of SSOM decoder.

(1) Initialization. Choose random values as initial weight vectors to $\mathrm{W}_{\mathrm{XN}}$ and $\mathrm{W}_{\mathrm{NY}}$. Note: self-organizing map decoder is symmetrical, that is:

$W_{N Y}^{\prime}[j][i]=W_{X N}[i][j] \quad i=1,2,3 \ldots n ; j=1,2,3 \ldots m$

(2) Calculate the threshold $d T$ according to Equation (2) and activating rate $\xi$ according to Equation (3).

$d T=\frac{1}{m n} \sum_{i=1}^{n} \sum_{j=1}^{m} W[i][j]$

$\xi=d T / 2$

(3) Accept an input codeword into the SSOM decoder.

(4) Similarity matching. Find the best-matching (winning) neuron node according to Equation (4). The specific equation is as follows:

$N e u=\arg \min _{j} \sum_{i=1}^{n}|X[i]-W[i][j]| \quad j=1,2,3 \ldots . m$

In the Equation (4), $m$ is the number of neuron node. $n$ is the dimension of input codeword. And only one neuron node is activated to respond an input vector (input codeword) at a time.

(5) Modification and output.

Modification: adjust the weight vectors to strengthen the activated neuron node (Equation (5)).

$$
\Delta W[i][j]=\xi *(X[i]-W[i][j]) \quad \text { if } j=\mathrm{Neu}
$$

In Equation (5), $\xi$ is activating rate calculated by Equation (3). Neu is the number of current activating neuron (winning neuron). In the decoder, input codeword is responded only by one neuron. And Equation (5) is used to reduce the distance between weight vector of activating neuron and current input codeword vector. As the decoder adopts symmetrical structure, the weight vectors' modification also is symmetrical. Therefore, the weight vectors be-
The Structure of Output Node

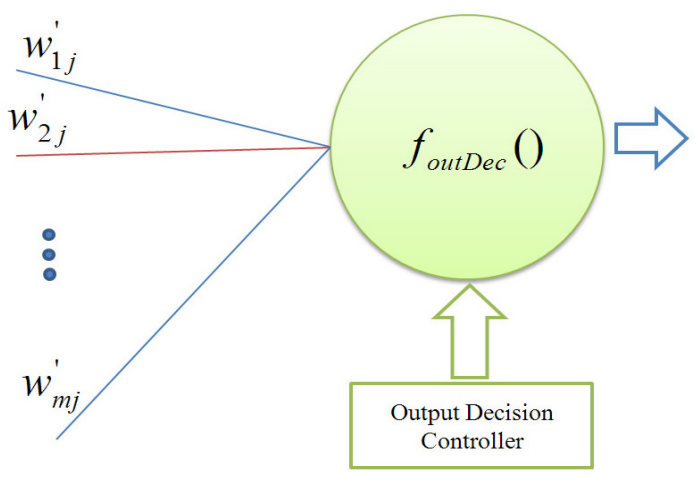

Fig. (2). The structure of output node.

tween neuron layer and output layer should be modified according to Equation (1) at the same time.

Output: get the ultimate result of current input codeword through output decision controller.

The output nodes (Fig. 2) accept activating node number from Output Decision Controller and choose corresponding weights (red $w^{\prime}{ }_{2 j}$ for example), and through function $f_{\text {outDec }}($ ) to make ultimate decision to output.

$$
f_{\text {outDec }}\left(W^{\prime}[i][j]\right)= \begin{cases}1 & \text { if } W^{\prime}[i][j] \geq d T \\ 0 & \text { if } W^{\prime}[i][j]<d T\end{cases}
$$

In Equation (6), $d T$ is an decision threshold of the decoder. The specific value of $d T$ depends on initial weight sets (Equation (2)).

\section{(6) Obtain the state of SSOM decoder}

If the weight change is small for all neurons, the SSOM is in steady phase at the time. Generally, the Equation (7) is the criterion to judge the state of SSOM. If all weight modifications meet the Equation (7), the decoder is in steady state, and the activating rate should be reduce according to Equation (8).

$\sum_{i=1}^{n}|\Delta W[i][j]|<d T / n \quad j=1,2,3 \ldots m$

$\xi=\xi / n$

(7) Continuation. Continue with step (3) to accept next codeword.

\section{PERFORMANCE ANALYSIS OF SSOM DECODER (LDPC FOR EXAMPLE)}

\subsection{Complexity Analysis}

LDPC code is a typical linear error correcting code, which is widely applied in free space optical (FSO) communication field. The complexity comparison between tradition decoder and SSOM decoder is implemented in this section.

Generally, the main computing complexity of the decoders is recorded in Table $\mathbf{1}$ below. 
Table 1. The main computing complexity of traditional decoder and SSOM decoder.

\begin{tabular}{|c|c|c|}
\hline Arithmetic Type & XOR & Addition \\
\hline \hline Traditional Decoder(Sum-Product Algorithm) & $k m n$ & \\
\hline SSOM Decoder & & $n x$ \\
\hline
\end{tabular}

$k$ is the iteration of tradition decoder. $m$ is the row number of check matrix. $n$ is the dimension of codeword. $x$ is the number of allowable codeword.

As for traditional decoder, $m$ and $n$ are generally determined in advance. $k$ is determined by the computing result between current codeword and check matrix, which mostly depends on the BER(bit error rate) of current input codeword. Therefore, the BER of input codeword is an important factor to impact the complexity of traditional decoder.

On the other hand, the complexity of SSOM decoder depends on the number of allowable codeword and dimension of codeword. Generally speaking, dimension of code word is fixed beforehand. Thus, the number of allowable codeword is a major factor to determine the complexity of SSOM decoder.

Form the analysis above, the complexity of SSOM decoder has nothing to do with the BER of input codeword. Naturally, both learning and decoding can be achieved instantly. However, the number of allowable codeword has directly influence on the computing complexity.

\subsection{Analysis of Simulation Result}

The section will discuss the decoding simulations of primitive LDPC code $(10,4)$. For the purpose of comparison, the BER of traditional decoder and SSOM decoder are simulated in different SNR(signal to noise ratio). Simulations results are shown in Fig. (3).

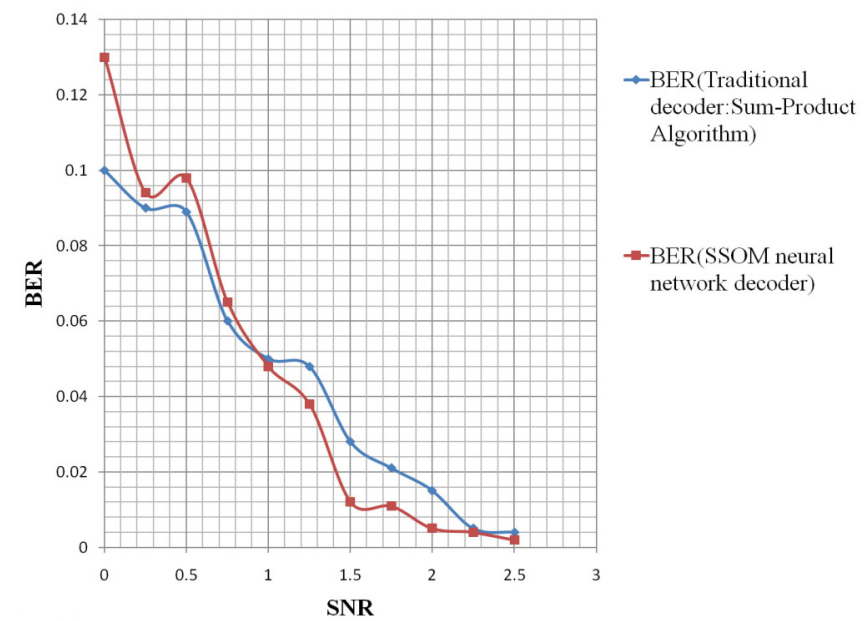

Fig. (3). Comparison between traditional decoder and SSOM decoder.

From the Fig. (3), traditional decoder has good performance when the SNR is low. However, the SSOM decoder has better performance than traditional decoder when the SNR is bigger than $1 \mathrm{Db}$.
Low SNR means more errors in the codeword sets. SSOM decoder belongs to neural network decoder. Learning from sample sets is a main characteristic. The initial weight sets cannot be matched with codeword perfectly at the beginning stage. Therefore, more mistakes occur in the condition of low SNR. However, the performance of SSOM decoder will be improved in steady phase.

On the other hand, learning from codeword sets is an intelligent process, which is like human learning. Therefore, higher possibility of the codeword emergence means greater probability of correct error correction.

\section{CONCLUSION}

In this paper, several contributions are achieved as follows:

(1) Construct a workable architecture of neural network decoder based on SSOM, which can achieve intelligent decoding function.

(2) Learning and decoding are achieved at the same time in the SSOM decoder, which has certain intelligence in comparison with traditional decoder.

(3) Rather than training the neural network by sample codeword sets in advance [5], the SSOM decoder can be adopted directly in communication system. Learning from the sample codeword sets is the major feature of the decoder, where the characteristic of sample codeword sets is extracted and stored in SSOM in distributed way.

(4) The SSOM neural network decoder can be adopted to decode any error correcting block codes without corresponding encoding rules.

(5) Compare to traditional error correcting decoder, it is easy to construct, more intelligent to different codeword sets.

On the other hand, the initial weight sets are random in SSOM decoder, which cannot match the codeword perfectly. Naturally, wrong decision generally occurs in the beginning stage. And the error will be gradually reduced with the decoder learning process. Therefore, it is essential to study the algorithm in initializing the weight sets of SSOM decoder.

Furthermore, when the number of allowable codeword increases, the computing complexity will increase gradually. Naturally, the SSOM decoder is suitable for lower number of allowable codeword.

Above all, when the allowable codeword is chosen randomly regardless of any encoding rule, only by learning from the codeword is the solution for decoding such codeword sets. SSOM decoder can be adopted in such condition, which is a kind of secure communication. Thus, SSOM decoder has certain prospect in private communication systems, which require higher reliability and security.

\section{CONFLICT OF INTEREST}

The authors confirm that this article content has no conflict of interest. 


\section{ACKNOWLEDGEMENTS}

This work was financially supported by Aviation Science Foundation of China (No. 201351S5002).

\section{REFERENCES}

[1] A.R. Karami, A.M. Ahmadian, and H. Tavakoli, "Multi layer perceptron neural networks decoder for LDPC codes", In: $5^{\text {th }}$ International Conference on Wireless Communications, Networking and Mobile Computing, 2009, pp. 1-4.

[2] S. M. Berber, and V. Kecman, "Convolutional decoders based on artificial neural networks", IEEE International Conference on Neural Networks - Conference Proceedings, vol. 2, pp. 1551-1556, 2004.

[3] H. Mohabeer, and K.M.S. Soyjaudah, "Application of predictive coding in the evolution of artificial neural network", In: $3^{\text {rd }}$ IEEE International Conference on Cognitive Infocommunications, 2012, pp. $775-780$.
[4] A. S. Hadi, "Linear block code decoder using neural network", In: Proceedings of the International Joint Conference on Neural Networks, 2008, pp. 1111-1114.

[5] R. Annauth, and H.C.S. Rughooputh, "Neural network decoding of turbo codes", IJCNN'99 vol. 5, pp. 3336-3341, 1999.

[6] J. Yuan, C. He, W. Gao, J. Lin, and Y. Pang, "A novel hard decision decoding scheme based on genetic algorithm and neural network", Optik - International Journal for Light and Electron Optics, vol. 125, no. 14, pp. 3457-3461, 2014.

[7] Q. N. Charei, H.F. Aghamalek, S. M. Razavi, and M. Golestanian, "An Improved soft decision method in Viterbi decoder using artificial neural networks", In: ${ }^{\text {st }}$ Iranian Conference on Pattern Recognition and Image Analysis, PRIA, 2013, pp. 1-4.

[8] M.E. Buckley, and S.B. Wicker, "A neural network for predicting decoder error in turbo decoders", IEEE Communications Letters, vol. 3, no. 5, pp. 145-147,1999.

[9] S. M. Berber, "A soft decision output convolutional decoder based on the application of neural networks", Proceedings - IEEE Military Communications Conference MILCOM, vol. 3, pp. 1495-1500, 2005.

Received: June 10, 2015

Revised: July 29, 2015

Accepted: August 15, 2015

(C) Xiao and Ai; Licensee Bentham Open.

This is an open access article licensed under the terms of the Creative Commons Attribution Non-Commercial License (http://creativecommons.org/licenses/by-nc/3.0/) which permits unrestricted, non-commercial use, distribution and reproduction in any medium, provided the work is properly cited. 